\title{
ACA [Cl] observations of the starburst galaxy NGC 253
}

\author{
M. Krips ${ }^{1}$, S. Martín ${ }^{2,3}$, K. Sakamoto ${ }^{4}$, S. Aalto ${ }^{5}$, T. G. Bisbas ${ }^{6,7}$, A. D. Bolatto ${ }^{8}$, D. Downes ${ }^{1}$, A. Eckart ${ }^{9}$, \\ Ch. Feruglio ${ }^{10}$, S. García-Burillo ${ }^{11}$, J. Geach ${ }^{12}$, T. R. Greve ${ }^{13}$, S. König ${ }^{5}$, S. Matsushita ${ }^{4}$, R. Neri ${ }^{1}$, S. Offner ${ }^{14}$, \\ A. B. Peck ${ }^{15}$, S. Viti ${ }^{13}$, and J. Wagg ${ }^{16}$ \\ 1 IRAM, Domaine Universitaire, 300 rue de la Piscine, 38406 Saint-Martin-d'Hères, France \\ e-mail: krips@iram.fr \\ 2 European Southern Observatory, Alonso de Córdova 3107, Vitacura, Santiago, Chile \\ 3 Joint ALMA Observatory, Alonso de Córdova 3107, Vitacura, Santiago, Chile \\ 4 Academia Sinica, Institute of Astronomy and Astrophysics, Taipei, Taiwan \\ 5 Department of Earth and Space Sciences, Onsala Observatory, Chalmers University of Technology, 43992 Onsala, Sweden \\ ${ }^{6}$ Max-Planck-Institut für Extraterrestrische Physik, Giessenbacherstr. 1, 85748 Garching, Germany \\ 7 Department of Astronomy, University of Florida, Gainsville, FL 32611, USA \\ 8 Department of Astronomy and Joint Space-Science Institute, University of Maryland, College Park, MD 20742, USA \\ 9 University of Cologne, I. Physikalisches Institut, Zülpicher Str. 77, 50937 Köln, Germany \\ 10 Scuola Normale Superiore, Piazza dei Cavalieri 7, 56126 Pisa, Italy \\ 11 Observatorio de Madrid (OAN-IGN), Alfonso XII, 3, 28014 Madrid, Spain \\ 12 Centre for Astrophysics Research, Science \& Technology Research Institute, University of Hertfordshire, Hatfield AL109AB, UK \\ 13 Department of Physics and Astronomy, UCL, Gower St., London, WC1E 6BT, UK \\ 14 Department of Astronomy, University of Massachusetts-Amherst, Amherst, MA, USA \\ 15 NRAO, 520 Edgemont Rd., Charlottesville, VA 22903, USA \\ 16 SKA Organziation, Lower Withington Macclesfield, Cheshire SK11 9DL, UK
}

Received 9 May 2016 / Accepted 24 June 2016

\begin{abstract}
Context. Carbon monoxide (CO) is widely used as a tracer of the molecular gas in almost all types of environments. However, several shortcomings of $\mathrm{CO}$ complicate usaging it as $\mathrm{H}_{2}$ tracer, such as its optical depth effects, the dependence of its abundance on metallicity, or its susceptibility to dissociation in highly irradiated regions. Neutral carbon emission has been proposed to overcome some of these shortcomings and hence to help revealing the limits of $\mathrm{CO}$ as a measure of the molecular gas.

Aims. We aim to study the general characteristics of the spatially and spectrally resolved carbon line emission in a variety of extragalactic sources and evaluate its potential as complementary $\mathrm{H}_{2}$ tracer to $\mathrm{CO}$.

Methods. We used the Atacama Compact Array to map the $[\mathrm{CI}]\left({ }^{3} \mathrm{P}_{1}-{ }^{3} \mathrm{P}_{0}\right)$ line emission in the nearby starburst galaxy NGC 253 at unprecedented angular resolution $\left(\sim 3^{\prime \prime}\right)$. This is the first well-resolved interferometric $[\mathrm{CI}]$ map of an extragalactic source.

Results. We have detected the [CI] line emission at high significance levels along the central disk of NGC 253 and its edges where expanding shells have previously been found in CO. Globally, the distribution of the $[\mathrm{CI}]$ line emission strongly resembles that of $\mathrm{CO}$, confirming the results of previous Galactic surveys that $[\mathrm{CI}]$ traces the same molecular gas as CO. However, we also identify a significant increase of $[\mathrm{CI}]$ line emission with respect to $\mathrm{CO}$ in (some of) the outflow or shocked regions of NGC 253, namely the bipolar outflow emerging from the nucleus. A first-order estimate of the $[\mathrm{CI}]$ column densities indicates abundances of $[\mathrm{CI}]$ that are very similar to the abundance of CO in NGC 253. Interestingly, we find that the $[\mathrm{CI}]$ line is marginally optically thick within the disk. Conclusions. The enhancement of the $[\mathrm{CI}] / \mathrm{CO}$ line ratios $(\sim 0.4-0.6)$ with respect to Galactic values $(\leq 0.1)$, especially in the shocked regions of NGC 253, clearly indicates that mechanical perturbation such as shocks and the strong radiation fields in this starburst galaxy have a marked effect on the carbon excitation and/or abundance.
\end{abstract}

Key words. galaxies: active - submillimeter: galaxies - galaxies: ISM - galaxies: starburst - galaxies: individual: NGC 253

\section{Introduction}

The feeding of, and feedback from, starbursts (SB) or supermassive black holes (AGN) in the central regions of galaxies are complex physical processes that vary with the evolutionary stage of and environmental conditions within the respective host galaxy. Detailed studies of the dynamics, spatial distribution, excitation conditions, and chemistry of the main constituents of the fueling material, the molecular, atomic, and ionized gas, are an indispensible tool for understanding the underlying physical mechanisms. The recent availability of ALMA's band 8 opens up a new era of studying the gas through neutral atomic carbon (C) emission in nearby galaxies (using its fine-structure lines $[\mathrm{CI}])$ at an unprecedented high angular and spectral resolution, an $\mathrm{H}_{2}$ tracer that is potentially comparable in strength to carbon monoxide (CO; e.g., Stutzki et al. 1997; Weißet al. 2003, 2005; Papadopoulos \& Greve 2004; Walter et al. 2011; Offner et al. 2014). Contrary to expectations from homogeneous cloud models (see, e.g., Hollenbach \& Tielens 1999), observations demonstrate that $\mathrm{C}$ is not only constrained to a layer interfacing the 


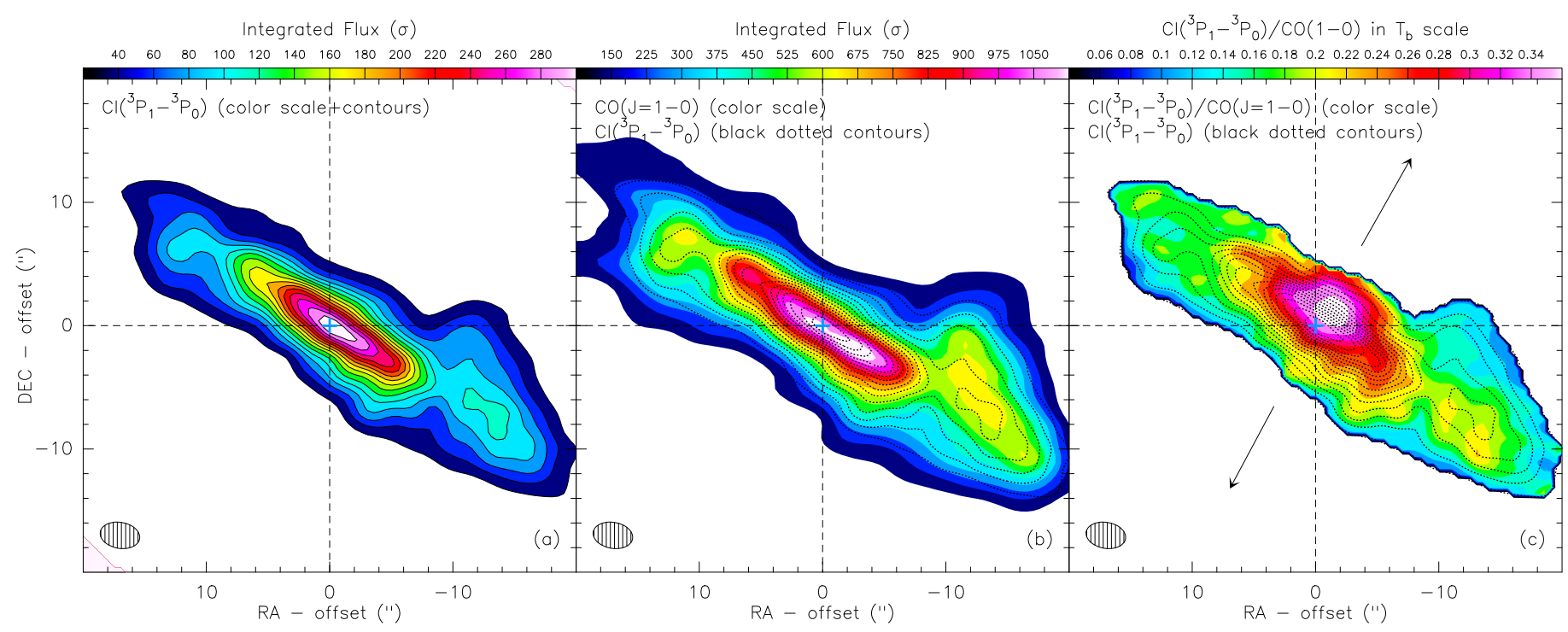

Fig. 1. Velocity-integrated maps of the $[\mathrm{CI}]\left({ }^{3} \mathrm{P}_{1}-{ }^{3} \mathrm{P}_{0}\right)$ line emission (color scale and contours in panel a)), and the $\mathrm{CO}(J=1-0)$ line emission of NGC 253 (color scale, panel b)). Panel c) shows the line ratio between the $[\mathrm{CI}]$ and $\mathrm{CO}$ emission in $T_{\mathrm{b}}$ scale (in color); to convert the line ratios from temperature scale into energy units (i.e., from $\mathrm{K} \mathrm{kms} \mathrm{s}^{-1}$ for the intensities to $\mathrm{W} \mathrm{m}^{-2} \mathrm{sr}^{-1}$ ), it needs to be multiplied by a factor of $\sim 80$ $\left(\equiv\left(v_{\mathrm{CI}(1-0)} / v_{\mathrm{CO}(1-0)}\right)^{3}\right)$. In all panels the contours represent [CI] line emission from panel a). Contours (and/or color steps) start from $40 \sigma$ to $300 \sigma$ in steps of $20 \sigma\left(\equiv 108 \mathrm{Jy} / \mathrm{beam} \mathrm{km} \mathrm{s} \mathrm{km}^{-1}=12 \mathrm{~K} \mathrm{~km} \mathrm{~s}^{-1}\right)$ for panel a), from $150 \sigma$ to $1200 \sigma$ in steps of $75 \sigma\left(\equiv 21.6 \mathrm{Jy} / \mathrm{beam} \mathrm{km} \mathrm{s}^{-1}=43 \mathrm{~K} \mathrm{~km} \mathrm{~s}{ }^{-1}\right)$ for panel b) (color steps), and from 0.04 to 0.36 in steps of 0.02 for panel c) (color steps). The crossing of the dashed lines in each panel marks the center of NGC 253: RA $=00^{\mathrm{h}} 47^{\mathrm{m}} 33^{\mathrm{s}} 17$ and Dec $=-25^{\circ} 17^{\prime} 17.1^{\prime \prime}$. The synthesized beam is shown as a dashed ellipse in the lower left corner of each panel. The arrows in panel c) indicate the direction of the outflows seen in X-ray and H $\alpha$ images (see, e.g., Bolatto et al. 2013).

ionized outer regions (dominated by ionized carbon) and the shielded cores (CO dominated), but one that is well mixed throughout the clouds (e.g., Keene et al. 1996; Ikeda et al. 2002; Shimajiri et al. 2013). This is thought to be a consequence of the timescale of several Myr for the chemical formation of $\mathrm{C}$ to $\mathrm{CO}$, which is similar to the dynamical timescales (see Suzuki et al. 1992; Lee et al. 1996) and density inhomogeneities created by turbulences (e.g., Glover et al. 2015). Especially in active environments, the dissociation of $\mathrm{C}$-bearing molecules such as $\mathrm{CO}$ by the strong radiation fields, either in the form of UV-radiation from an SB, X-rays from an AGN, or cosmic rays from a supernova as well as mechanical processes through shocks, can significantly enhance the $\mathrm{C}$ abundance (e.g., Hollenbach \& Tielens 1999; Tanaka et al. 2011; Meijerink et al. 2011; Bisbas et al. 2015). Single-dish observations have demonstrated that $[\mathrm{CI}] / \mathrm{CO}$ ratios are significantly higher in active environments than in Galactic clouds (e.g., Stutzki et al. 1997; Israel et al. 1995; Israel 2009; Walter et al. 2011). Among others, the low critical densities $\left(n_{\text {crit }}=3 \times 10^{3} \mathrm{~cm}^{-3}\right.$; e.g., Kaufman et al. 1999) of its ground-state line makes $\mathrm{C}$ as easily excited as $\mathrm{CO}$ in molecular clouds. All this promotes $\mathrm{C}$ as a complementary $\mathrm{H}_{2}$ tracer to $\mathrm{CO}$, especially in active galaxies. However, the possible "superiority" of $\mathrm{C}$ to $\mathrm{CO}$, as a tracer of $\mathrm{H}_{2}$, has been questioned by Israel et al. (2015), who found a poor correlation in a large survey of Ultra-Luminous Infra-Red Galaxies (ULIRGs). The [CI] line emission in their sources seems to be mostly associated with dense and not with diffuse gas and depends strongly on the excitation conditions.

This is the first publication in a series of $\mathrm{C}$ studies presenting observations of high angular resolution and high sensitivity of the $[\mathrm{CI}]$ emission in nearby active galaxies. In this Letter, we concentrate on the nearby $\left(3.5 \mathrm{Mpc}\right.$, where $1^{\prime \prime}=$ 17 pc (see Mouhcine et al. 2005; Rekola et al. 2005) starburst galaxy NGC 253 (for more details see Sakamoto et al. 2011; Bolatto et al. 2013; Krips et al. 2016).

\section{Observations}

The observations of the neutral atomic carbon fine-structure line emission $\left([\mathrm{CI}]\left({ }^{3} \mathrm{P}_{1}-{ }^{3} \mathrm{P}_{0}\right)\right.$ in the nearby starburst galaxy NGC 253 were carried out on July 262015 with the Atacama Compact Array (ACA) as part of the ALMA project 2013.1.00368.S in Cycle 2 . The line transition is at a rest frequency of $492.161 \mathrm{GHz}$, and we refer to it as [CI](1-0) in the following. No ALMA $12 \mathrm{~m}$ array observations were carried out because of weather constraints, but the ACA observations are of such a good quality and angular resolution by themselves that they warrant publication.

The ACA observations were carried out with 11 antennas that were spaced at uv-distances ranging between $\sim 8 \mathrm{~m}$ up to $50 \mathrm{~m}$ and hence recovering large-scale structures up to $\sim 15^{\prime \prime}$. This gives a synthesized beam of 3 ".24 $\times 2$ '!11 at a position angle of 80 degrees at $492 \mathrm{GHz}$ (natural weighting). In total, 22 mosaic fields were placed along the central $\sim 80^{\prime \prime} \times 80^{\prime \prime}$ of NGC 253; the field of view of each individual mosaic field amounts to $\sim 21^{\prime \prime}$. The on-source time corresponds to three hours in total, split into eight to ten minutes per field. Four spectral windows were centered on the following observed frequencies: $491.772 \mathrm{GHz}([\mathrm{CI}](1-0))$ and $489.905 \mathrm{GHz}$ in the upper sideband, and $479.521 \mathrm{GHz}$ and $477.884 \mathrm{GHz}$ in the lower sideband, with an initial spectral resolution of $1.9053 \mathrm{MHz}\left(\equiv 1.19 \mathrm{~km} \mathrm{~s}^{-1}\right)$ and a total bandwidth of $1.99 \mathrm{GHz}$ each. The data were subsequently sampled to a lower spectral resolution of $4.88 \mathrm{MHz}$ $\left(\equiv 2.98 \mathrm{~km} \mathrm{~s}^{-1}\right)$. We used Neptune as absolute flux, J2253+1608 as bandpass, and J0038-2459 as gain (amplitude and phase-intime) calibrators. There were no signs of line emission or absorption in Neptune in any of the observed spectral windows, and we used the planetary model defined in the Butler-JPL- Horizons 2012 catalog. Average system temperatures were between 300 and $600 \mathrm{~K}$ at $480 \mathrm{GHz}-490 \mathrm{GHz}$. We reached an rms noise level of $\sim 150 \mathrm{mJy}$ in $3 \mathrm{~km} \mathrm{~s}^{-1}$ wide velocity channels for the spectral window centered on $492 \mathrm{GHz}$. We reduced the data with the 
ALMA CASA package (version 4.3.1; McMullin 2007) but performed subsequent processing with the GILDAS package ${ }^{1}$.

The continuum emission was derived from all line-free channels (i.e., excluding the $[\mathrm{CI}]$ and the marginally detected $\mathrm{CS}$ line emission) and subsequently subtracted from the uv-visibilities in all channels to create continuum-free line data cubes. The positions agree well with those extracted from the $\mathrm{CO}(J=1-0)$ data cube, indicating accurate astrometry for both data sets within the errors.

ALMA 12 m array cycle 0 observations are available for the $\mathrm{CO}(J=1-0)$ line emission from the ALMA science archive (2011.0.00172.S; see Bolatto et al. 2013). The compact configuration used for these observations corresponds well to the scales covered with the ACA $[\mathrm{CI}]$ observations of $\leq 20^{\prime \prime}$; the spectrally resolved line emission shows scales of $\leq 10^{\prime \prime}$ so that no different resolution effects are expected. Given also the excellent uvcoverage, we used a slight uv-taper to smooth the initial angular resolution to that of our $[\mathrm{CI}](1-0)$ observations.

\section{Results and discussion}

The $[C I](1-0)$ line emission is clearly detected throughout the circumnuclear disk of NGC 253. In both peak location and distribution, it strongly resembles the $\mathrm{CO}$ line emission (see Fig. 1 as well as Sakamoto et al. 2011; Bolatto et al. 2013; Krips et al. 2016). The $[C I](1-0)$ line emission shows the strongest peak at what is believed to be the center of NGC 253 (see Turner \& Ho 1985; Sakamoto et al. 2011; Müller-Sánchez et al. 2010), which also coincides with one of the (sub-)millimeter continuum and $\mathrm{CO}$ line peaks. Compared to single-dish $[\mathrm{CI}](1-0)$ line emission spectra observed in NGC 253 (Israel et al. 1995), we have probably resolved out less than $20 \%$ of the $[\mathrm{CI}](1-0)$ line emission in the central $\sim 50^{\prime \prime}$ with our ACA observations. This is on the order of the absolute flux calibration uncertainties between the two observations.

The spectrally integrated line ratios between the $[\mathrm{CI}](1-0)$ and $\operatorname{CO}(J=1-0)$ line emission are plotted in brightness temperature scale in Fig. 1c. The line ratios are mostly $\sim 0.2-0.3$ within the disk with a very pronounced absolute maximum of almost 0.4 , which is located a few arcseconds NW of the central part of the disk. X-ray, $\mathrm{H} \alpha$ and $\mathrm{CO}(J=1-0)$ emission maps reveal a strong outflow in the same NW direction (see Bolatto et al. 2013). To assess whether the spectrally integrated maps hide further details and/or velocity-dependent structures, we derived position-velocity diagrams for the $[\mathrm{CI}](1-0)$ and $\mathrm{CO}(J=1-0)$ line emission along and perpendicular to the disk in NGC 253 (see Figs. 2a and c). We also calculated the line ratios in temperature scale for the two velocity slices (see Figs. $2 b$ and d). In addition to the pronounced global maximum toward the NW that is only marginally captured with the chosen slice along the disk we find an even stronger local line ratio peak of almost 0.6 that is located between the center and the western edge of the disk. It extends over roughly $50 \mathrm{~km} \mathrm{~s}^{-1}$ so it is averaged out in the line ratio map of Fig. 1, which was spectrally integrated over a much wider velocity range $\left(\sim 400 \mathrm{~km} \mathrm{~s}^{-1}\right)$. Figure 3 shows the channel maps of the line ratios between velocities of $-40 \mathrm{~km} \mathrm{~s}^{-1}$ and $-94 \mathrm{~km} \mathrm{~s}^{-1}$ to indicate where this line ratio peak is located within the disk and how it behaves dynamically. Although Fig. 1 suggests that the higher $[\mathrm{CI}](1-0) / \mathrm{CO}(J=1-0)$ line ratio only extends in NW direction perpendicular to the disk, the region of the highest ratios is also extended within the disk in the western as well as south-eastern direction (Fig. 2). The outflow is known to

\footnotetext{
1 http://wWw.iram. fr/IRAMFR/GILDAS
}

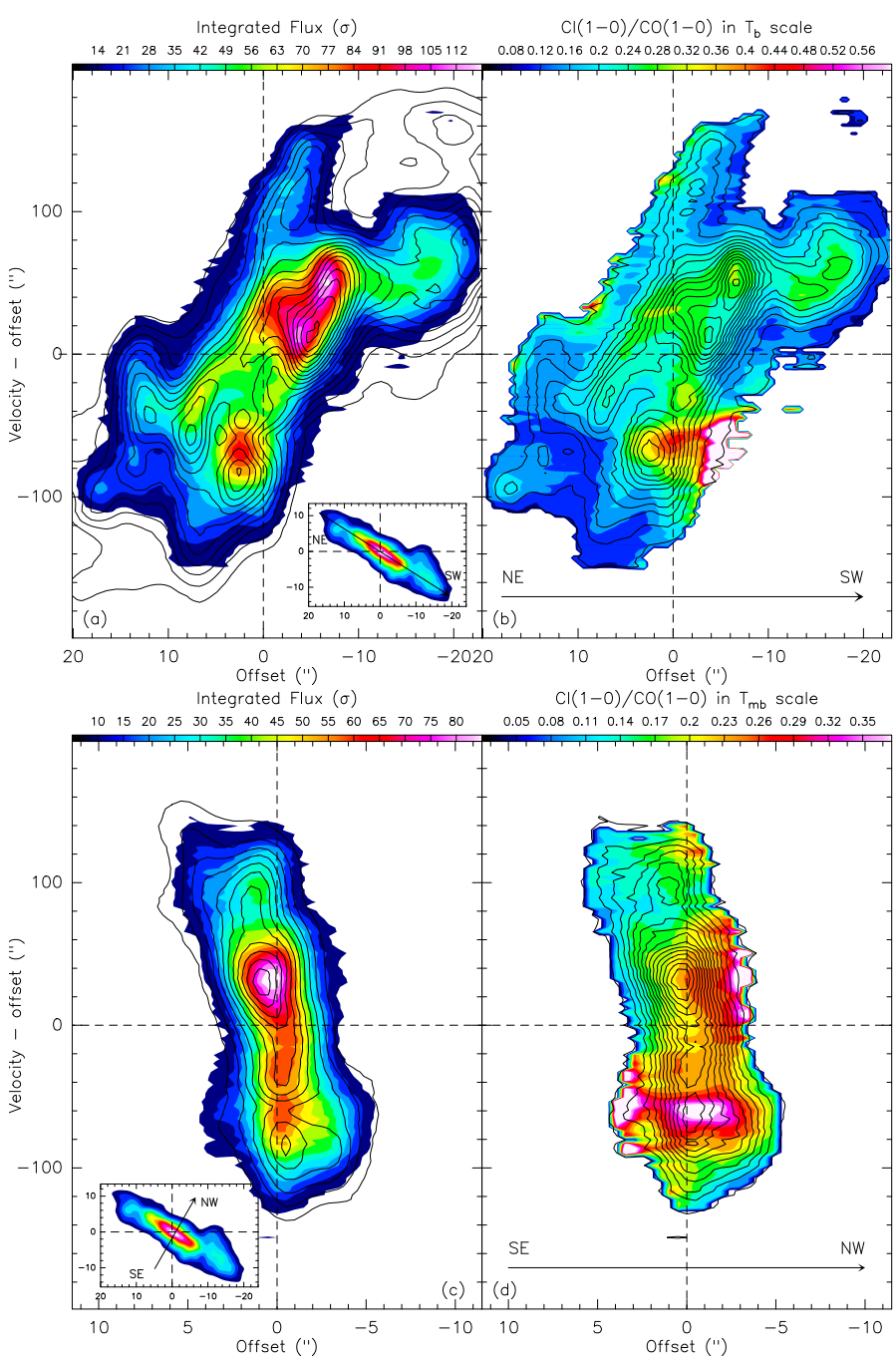

Fig. 2. Position-velocity diagram of the $[\mathrm{CI}](1-0)$ line emission (color scale) taken along the disk (upper panels $a+b$ ) and perpendicular to it (lower panels $c+d$; the slices are chosen such as that they go through the maximum peak of the line ratio map shown in Fig. 1c). The $\mathrm{CO}(J=$ 1-0) line emission (contours) is overlaid on that of $[\mathrm{CI}]$ in panels a) and c); the velocity resolution is $3 \mathrm{~km} \mathrm{~s}^{-1}$. Panels b) and d) show the $[\mathrm{CI}](1-0) / \mathrm{CO}$ line ratio map in brightness temperature scale overlaid with contours of the $[\mathrm{CI}]$ emission. The contours of the $\mathrm{CO}(J=1-0)$ line emission are in steps of $30 \sigma(1 \sigma=7.4 \mathrm{mJy} /$ beam $)$ in panel a) and c) and that of the $[\mathrm{CI}]$ emission goes in steps of $7 \sigma(1 \sigma=150 \mathrm{mJy} / \mathrm{beam})$ in panel $\mathbf{b}$ ) and in steps of $5 \sigma$ in panel d). The zero velocity corresponds to the kinematic LSR velocity of $236 \mathrm{~km} \mathrm{~s}^{-1}$.

be bipolar (see Bolatto et al. 2013), therefore we would expect to find similar ratios on both sides of the outflow if the shock itself were the cause of this phenomenon. The extension to the west is less clear, although an interaction might be taking place between the central outflow and the western edge where an expanding shell is suspected (see Sakamoto et al. 2011; Bolatto et al. 2013; Krips et al. 2016). Although we see a bipolarity of the increased line ratios in the velocity-resolved maps, we cannot completely exclude a possible shadowing (i.e., optical thickness effect) of the $\mathrm{CO}(J=1-0)$ line emission in the NW part of the outflow by the disk whose near-side is the NW part (see Sakamoto et al. 2006,2011 ) because of the asymmetry in the line ratios between the NW and SE parts. The shadowing by the disk could artificially decrease the $\operatorname{CO}(J=1-0)$ emission and hence increase the $[\mathrm{CI}](1-0) / \mathrm{CO}(J=1-0)$ line ratio there. However, the fact 


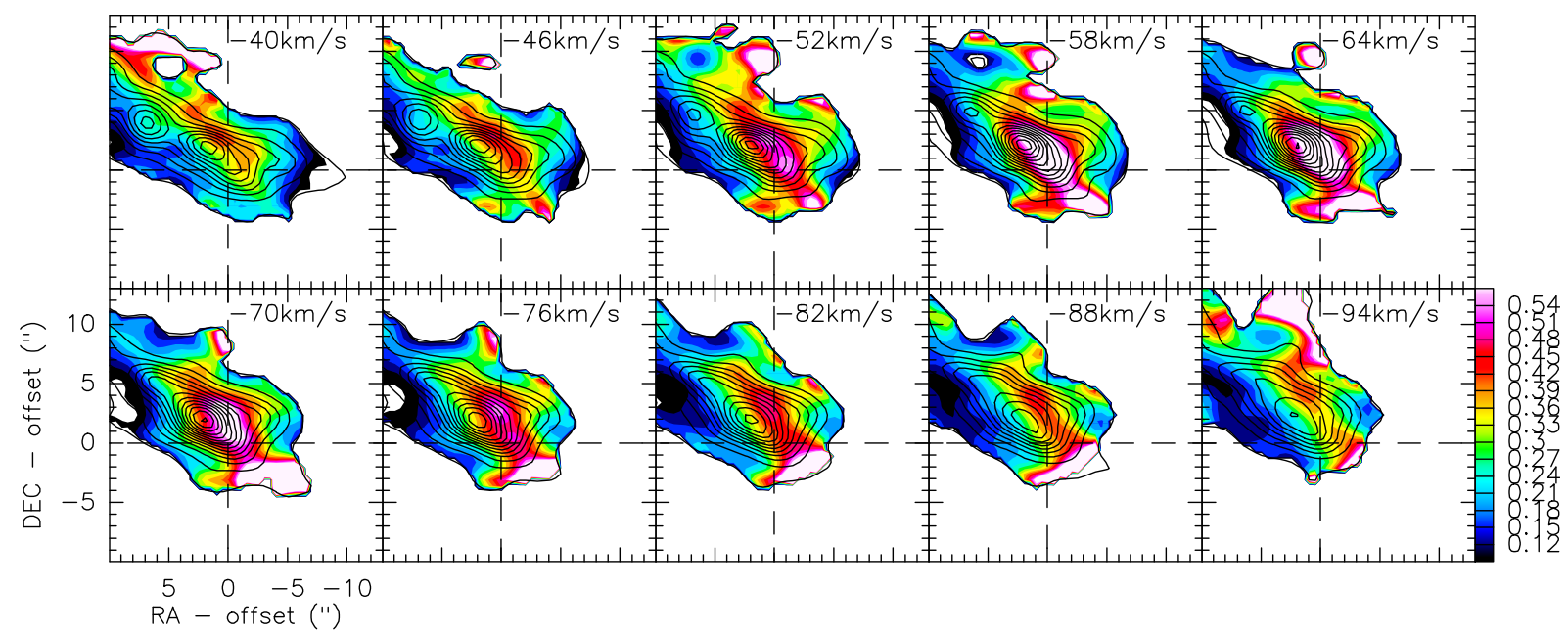

Fig. 3. Channel maps of the $[\mathrm{CI}](1-0) / \mathrm{CO}(J=1-0)$ line ratios in $T_{\mathrm{mb}}$-scale (color scale) between velocities $-40 \mathrm{~km} \mathrm{~s}^{-1}$ and $-94 \mathrm{~km} \mathrm{~s}^{-1}$ overlaid with the integrated $[\mathrm{CI}](1-0)$ line emission (contours); the color scales starts from 0.09 and go in steps of 0.3 , the contours of [CI](1-0) start at $5 \sigma$ and go in steps of $10 \sigma(1 \sigma=106 \mathrm{mJy} /$ beam $)$. Note that the velocity resolution has been resampled to $6 \mathrm{~km} \mathrm{~s}^{-1}$.

that we see a similar though less pronounced trend in the line ratios toward the SE part of the outflow probably indicates that the shadowing might only magnify the effect in the NE part, which would explain the asymmetry of this bipolarity. Furthermore, the findings by Krips et al. (2016) suggest that the high $[\mathrm{CI}](1-0) / \mathrm{CO}(J=1-0)$ ratio of $\gtrsim 0.4$ is not due to a temperature and hence a less excited $\mathrm{CO}(J=1-0)$ line must be due to an abundance effect (either in $\mathrm{C}$ and/or $\mathrm{CO}$ ). A very early paper on $[\mathrm{CI}](1-0)$ emission by Little et al. (1998) has reported on higher $[\mathrm{CI}](1-0) / \mathrm{CO}(J=1-0)$ ratios of $\simeq 0.4$ in the bipolar outflow of the Galactic molecular cloud G5.2-0.74N. The authors argued that the $\mathrm{CO}$ emission had been dissociated by far-UV radiation from the central source in the cavity of the outflow, hence reducing the $\mathrm{CO}$ abundance and probably at the same time increasing that of C (see also Offner et al. 2014; Tanaka et al. 2011).

The simulations by Kaufman et al. (1999) showed that the $\mathrm{CI}[1-0] / \mathrm{CO}(J=1-0)$ line ratios increase with decreasing densities in photon-dominated regions (PDR). For the line ratios of 0.2-0.3 along the disk, densities of $\sim 10^{4} \mathrm{~cm}^{-3}$ predicted by their models are mostly consistent with those calculated from previous LVG simulations in NGC 253 (e.g., Krips et al. 2016); the disk in NGC 253 is thought to be dominated by PDRs (e.g., Martín et al. 2009). If the entire gas emission in NGC253 is simply due to PDR excitation or chemistry alone, the increase to $0.5-0.6$ to the NW and SE found in our observations might then be a simple density effect. However, if this were true, we would expect higher ratios toward the eastern part of the disk as well, that is, everywhere where we expect the density to fall, but this is not the case. Moreover, the good agreement between the peak of the line ratio and the outflow from the disk imply that shocks could be an additional factor for the increased line ratios here.

\subsection{Opacities, column densities, and $\mathrm{C} / \mathrm{CO}$ abundance ratios}

We cannot a priori assume optically thin emission for the $[\mathrm{CI}](1-0)$ line emission, therefore we start by estimating its optical depth. Following the approach used by Ikeda et al. (2002) and Glover et al. (2015), we estimated the [CI](1-0) opacity from the excitation temperature derived from the optically thick $\mathrm{CO}(J=1-0)$ line emission as a first approximation, assuming that the excitation temperatures are identical for $\mathrm{CO}$ and $\mathrm{C}$ and that they coexist in the same gas regions. We find excitation temperatures of $T_{\mathrm{ex}, \mathrm{CO}} \simeq 7-13 \mathrm{~K}\left(\equiv T_{\mathrm{ex}, \mathrm{C}}\right)$ along the disk of NGC 253, which is consistent with the range stated in Meier et al. (2015), and [CI](1-0) opacities of $\tau_{[\mathrm{CI}]} \simeq 0.8-0.9$. The $[\mathrm{CI}](1-0)$ line emission is marginally optically thick. This translates into an optical depth correction factor of $f_{\tau} \simeq 1.5$ that has to be applied to the carbon column densities $\left(f_{\tau}=1\right.$ corresponds to the optically thin case). Using the specific parameters for the $[\mathrm{CI}](1-0)$ line such as the Einstein A coefficient of $7.9 \times 10^{-8} \mathrm{~s}^{-1}$ (see Nussbaumer 1971) and integrated fluxes of 300 to $1620 \mathrm{Jy} /$ beam $\mathrm{km} \mathrm{s}^{-1}\left(3^{\prime \prime} .2 \times 2^{\prime \prime} .1 \equiv(51 \times 34) \mathrm{pc}\right)$ along the disk of NGC 253, we find column densities of $N_{\mathrm{C}}=f_{\tau} \times 1.429 \times$ $10^{15} \int \mathrm{S}(\mathrm{Jy}) \mathrm{d} v /\left[\mathrm{Jy} \mathrm{km} \mathrm{s}^{-1}\right] \mathrm{cm}^{-2} \simeq(1-3) \times 10^{18} \mathrm{~cm}^{-2}$ (for a more detailed description of the formulae see Ikeda et al. 2002). These carbon column densities are significantly higher by a factor of 5-10 than those found in the Galactic center and several Galactic star forming molecular clouds (e.g., Tanaka et al. 2011; Plume et al. 1999) and lie at the upper end of the values found for turbulent molecular cloud models (Glover et al. 2015). They are also similar to the (averaged) column densities of $N_{\mathrm{CI}} / \mathrm{d} v \simeq 10^{18} \mathrm{~cm}^{-2} /\left(\mathrm{km} \mathrm{s}^{-1}\right)$ derived for the $\mathrm{C}$ survey of (U)LIRGs by Israel et al. (2015), who used a large-velocitygradient (LVG) modeling and single-dish observations.

Krips et al. (2016) performed an LVG analysis using several $\mathrm{CO}$ transitions and a two-phase temperature model of the molecular gas to determine CO column densities $N_{\mathrm{CO}}$ of a few times $10^{18} \mathrm{~cm}^{-2}$ (see also Meier et al. 2015). The best-fit CO column densities then indicate a $N_{\mathrm{CI}} / N_{\mathrm{CO}}$ ratio, or more directly, an abundance ratio $[\mathrm{C}] /[\mathrm{CO}]$ of $\sim 0.5-1$, which is higher by a factor of $\sim 5-10$ than the average found in Israel et al. (2015), but within their range for all galaxies. Thus, $\mathrm{C}$ seems to be almost as abundant as CO in NGC 253.

\section{Summary and conclusions}

Significant $[\mathrm{CI}](1-0)$ emission is detected in the central $\sim 1 \mathrm{kpc}$ of the starburst galaxy NGC 253 with the following results:

1. The $[\mathrm{CI}](1-0)$ line emission globally correlates well with that of $\operatorname{CO}(J=1-0)$, which supports the results from previous carbon studies of Galactic sources.

2. Globally, the $[\mathrm{CI}](1-0) / \mathrm{CO}$ line ratios are about $0.1-0.4$ in temperature scale and are therefore higher than those 
in the Milky Way, where values of $\leq 0.1$ are found (e.g., Wright et al. 1991).

3 . The $[\mathrm{CI}](1-0)$ line emission appears to be enhanced compared to $\mathrm{CO}(J=1-0)$ in a NW-SE direction perpendicular to the disk (along the bipolar outflow that emerges from the nucleus of NGC 253) and in the western region of the disk where outflows or shocks are important dynamical players. Galactic cloud outflows also exhibit an increase in the [CI]/CO line ratio (see Little et al. 1998; Tanaka et al. 2011). This strongly indicates that shocks and their associated radiation affect the $\mathrm{C}$ and/or $\mathrm{CO}$ excitation or abundance. However, other explanations cannot be excluded as yet, such as cosmic rays (e.g., Bisbas et al. 2015; Bradford et al. 2003), optical-depth effects, and low-density PDRs (Kaufman et al. 1999; see also discussion in Tanaka et al. 2011).

4. Based on a first-order estimate of the $[\mathrm{CI}]$ column densities, we find carbon abundances to be almost equal to those of $\mathrm{CO}$ within the central disk of NGC 253. Moreover, the $[\mathrm{CI}](1-0)$ line emission shows opacities of around $0.8-0.9$ that are not quite compatible with an optically thin gas. This agrees well with the findings by Israel et al. (2015) that $\mathrm{C}$ is mostly found in dense and not in diffuse gas.

Acknowledgements. K.S. was supported by the MOST grant 1092-2119-M001011-MY3, C.F. from PRIN MIUR 2010-2011, project "The Chemical and Dynamical Evolution of the Milky Way and Local Group Galaxies", prot. 2010LY5N2T, S.G.B. from MINECO grants AYA2013-42227-P and AYA2012 32295, S.O. from NSF grant AST-1510021, A.D.B. from NSF-AST1412419, and SM by the Ministry of Science and Technology of Taiwan with MoST 103 2112-M001-032-MY3.

\section{References}

Bisbas, T. G., Papadopoulos, P. P., \& Viti, S. 2015, ApJ, 803, 37 Bolatto, A. D., Warren, S. R., Leroy, A. K., et al. 2013, Nature, 499, 450 Bradford, C. M., Nikola, T., Stacey, G. J., et al. 2003, ApJ, 586, 891
Glover, S. C. O., Clark, P. C., Micic, M., \& Molina, F. 2015, MNRAS, 448, 1607 Hollenbach, D. J., \& Tielens, A. G. G. M. 1999, Rev. Mod. Phys., 71, 173 Ikeda, M., Oka, T., Tatematsu, K., Sekimoto, Y., \& Yamamoto, S. 2002, ApJS, 139,467

Israel, F. P. 2009, A\&A, 493

Israel, F. P., White, G. J., \& Baas, F. 1995, A\&A, 302, 343

Israel, F. P., Rosenberg, M. J. F., \& van der Werf, P. 2015, A\&A, 578, A95

Kaufman, M. J., Wolfire, M. G., Hollenbach, D. J., \& Luhman, M. L. 1999, ApJ, 527,795

Keene, J., Lis, D. C., Phillips, T. G., \& Schilke, P. 1996, IAU, 178, 129

Krips, M., Martín, S., Peck, A., et al. 2016, ApJ, 821, 112

Lee, H.-H., Herbst, E., Pineau des Forets, G., Roueff, E., \& Le Bourlot, J. 1996, A\&A, 311, 690

Little, L. T., Kelly, M. L., \& Murphy, B. T. 1998, MNRAS, 294, 105

Martín, S., Martín-Pintado, J., \& Viti, S. 2009, ApJ, 706, 1323

Meier, D. S., Walter, F., Bolatto, A. D., et al. 2015, ApJ, 801, 63

Meijerink, R., Spaans, M., Loenen, A. F., \& van der Werf, P. P. 2011, A\&A, 525, A119

McMullin, J. P., Waters, B., Schiebel, D., Young, W., \& Golap, K. 2007, Astronomical Data Analysis Software and Systems XVI, eds. R. A. Shaw, F. Hill, \& D. J. Bell (San Francisco, CA: ASP), ASP Conf. Ser., 376, 127

Mouhcine, M., Ferguson, H. C., Rich, R. M., Brown, T. M., \& Smith, T. E. 2005 ApJ, 633, 810

Müller-Sánchez, F., González-Martín, O, Férnandez-Ontiveros, J. A., Acosta-Pulido, J. A., \& Prieto, M. A. 2010, ApJ, 716, 1166

Nussbaumer, H. 1971, ApJ, 166, 411

Offner, S. S. R., Bisbas, Th., Bell, T. A., \& Viti, S. 2014, MNRAS, 440, 81 Papadopoulos, P., \& Greve, T. 2004, ApJ, 615, L29

Plume, R., Jaffe, D. T., Tatematsu, K., \& Evans, N. J. 1999, ApJ, 512, 768

Rekola, R., Richer, M. G., McCall, M. L., et al. 2005, MNRAS, 361, 330

Sakamoto, K., Ho, P. T. P., Iono, D., et al. 2006, ApJ, 636, 685

Sakamoto, K., Mao, R.-Q., Matsushita, S., et al. 2011, ApJ, 735, 19

Shimajiri, Y., Sakai, T., Tsukagoshi, T., et al. 2013, ApJ, 774, L20

Stutzki, J., Graf, U. U., Haas, S., et al. 1997, ApJ, 477, L33

Suzuki, H., Yamamoto, S., Ohishi, M., et al. 1992, ApJ, 392, 551

Tanaka, K., Oka, T., Matsumura, S., et al. 2011, ApJ, 743, L39

Turner, J. L., \& Ho, P. T. P. 1985, ApJ, 299, L77

Walter, F., Weiß, A., Downes, D., et al. 2011, ApJ, 730, 18

Weiß, A., Henkel, C., Downes, D., et al. 2003, A\&A, 409, L41

Weiß, A., Downes, D., Henkel, C., \& Walter, F. 2005, A\&A, 429, L25

Wright, E. L., Mather, J. C., Bennett, C. L., et al. 1991, ApJ, 381, 200 